\title{
Knowledge, Practice and Attitude Toward COVID-19 Among Physicians: Cross-Sectional Study.
}

\author{
Saddam Al Demour ${ }^{1}$, Mera ababneh ${ }^{2}$, Raed Al-Taher ${ }^{1}$, Adel Alrabadi ${ }^{1}$, Ahmad Jaradat ${ }^{3}$, \\ Faris Abushamma ${ }^{3}$, Fadi Al-Hadidi ${ }^{1}$, Baeth Al-Rawashdeh ${ }^{1}$, Mohammad Ihmeidan ${ }^{1}$, \\ Ahmad Abubaker ${ }^{4}$, and Mohammad Al-Zubi ${ }^{5}$ \\ ${ }^{1}$ The University of Jordan Faculty of Medicine \\ ${ }^{2}$ Jordan University of Science and Technology Faculty of Pharmacy \\ ${ }^{3}$ An-najah National University Faculty of Medicine and Health Sciences \\ ${ }^{4}$ Jordan University of Science and Technology Faculty of Medicine \\ ${ }^{5}$ Yarmouk University Faculty of Medicine
}

May 28, 2020

\begin{abstract}
Abstract: Aim: Coronavirus disease-2019 is an emerging disease that is threatening the world with a rapid increase in cases and deaths since it was first identified in December 2019. Our study aimed to assess the knowledge, practice, and attitude toward COVID-19 among physicians in Jordan and Palestine. Method: This is a cross-sectional study using an online survey conducted in April, 2020. A 36-items survey was developed and invitations were sent to physicians in different health sectors using social media. Results: A total of 454 physicians participated in this study with a mean age 36.2 ? 10.8 years. The mean score of basic knowledge was 4.4 ? 0.8 , and there were significant statistical differences between basic knowledge mean scores among physicians in different professional degrees and physicians in different health sectors $(\mathrm{P}=0.0315, \mathrm{P}=0.0137$ respectively $)$. Participants showed appropriate practical measures toward COVID-19, where the mean scores of indications for PCR test, indications for home quarantine, self-protection measures, and measures if physician self-suspected of COVID-19 were 5.4 ? $1.4,5.0$ ? 1.0, 6.1 ? 1.1, 9.9? 1.1 respectively. The mean score of attitudes toward COVID-19 were 41.5 ? 3.3 and significantly related to the age and different experience years $(\mathrm{P}=0.0022, \mathrm{P}=0.0077$ respectively). Conclusion: As the global threat of COVID-19 continues to emerge, it is critical to improve the knowledge, practice, and attitudes of physicians worldwide.
\end{abstract}

Title : Knowledge, Practice and Attitude Toward COVID-19 Among Physicians: Cross-Sectional Study.

Short title : Knowledge, Practice and Attitude Toward COVID-19

${ }^{1}$ Saddam Al Demour, ${ }^{2}$ Mera Ababneh, ${ }^{3}$ Raed Al-Taher, ${ }^{1}$ Adel Alrabadi, ${ }^{4,5}$ Ahmad Jaradat, ${ }^{4,5,6}$ Faris Abushamma, ${ }^{7}$ Fadi Al-Hadidi, ${ }^{8}$ Baeth Al-Rawashdeh, ${ }^{1}$ Mohammad Ihmeidan, ${ }^{9}$ Ahmad Abubaker, ${ }^{10}$ Mohammad Al-Zubi.

${ }^{1}$ Department of Special Surgery, Division of urology, School of Medicine, The University of Jordan, Amman 11942, Jordan.

2 Department of Clinical Pharmacy, Jordan University of Science and Technology, Faculty of Pharmacy, Irbid 22110 Jordan.

${ }^{3}$ Department of General Surgery, Division of Pediatric Surgery, School of Medicine, The University of Jordan, Amman 11942, Jordan. 
${ }^{4}$ Department of Medicine, Faculty of medicine and health Sciences, An-Najah National University, Nablus 44833, Palestine.

${ }^{5}$ Department of urology, An-Najah National University Hospital, Nablus 44833, Palestine.

${ }^{6}$ Bristol Urological Institute, North Bristol NHS Trust, Bristol, United Kingdom

7 Department of Special Surgery, Division of Orthopedics, School of Medicine, The University of Jordan, Amman 11942, Jordan.

8 Department of Special Surgery, Division of Otorhinolaryngology, School of Medicine, The University of Jordan, Amman 11942, Jordan.

${ }^{9}$ Department of Anesthesia, Jordan University of Science and Technology, Faculty of Medicine, Irbid 22110, Jordan.

${ }^{10}$ Department of Urology, Yarmouk University, Faculty of Medicine, Irbid 21163, Jordan

corresponding author:

Saddam Al Demour

Department of Special Surgery, Division of urology, The University of Jordan, School of Medicine, Amman 11942, Jordan.

Telephone: +962799144000

Orcid ID: https://orcid.org/0000-0003-4044-0927

Email: saldemour@ju.edu.jo

\section{Hosted file}

Manuscript with title, abstract,main text, tables.docx available at https://authorea.com/ users/327328/articles/454903-knowledge-practice-and-attitude-toward-covid-19-amongphysicians-cross-sectional-study 